\title{
A finite mass based method for Vlasov-Poisson simulations
}

\author{
David J. Larson ${ }^{\mathrm{a}, *}$, Christopher V. Young ${ }^{\mathrm{b}}$ \\ ${ }^{a}$ AX Division, Lawrence Livermore National Laboratory, Livermore, CA 94550, USA \\ ${ }^{b}$ Stanford Plasma Physics Laboratory, Stanford University, Stanford, CA 94305, USA
}

\begin{abstract}
A method for the numerical simulation of plasma dynamics using discrete particles is introduced. The shape function kinetics (SFK) method is based on decomposing the mass into discrete particles using shape functions of compact support. The particle positions and shape evolve in response to internal velocity spread and external forces. Remapping is necessary in order to maintain accuracy and two strategies for remapping the particles are discussed. Numerical simulations of standard test problems illustrate the advantages of the method which include very low noise compared to the standard particle-in-cell technique, inherent positivity, large dynamic range, and ease of implementation.
\end{abstract}

Keywords: plasma simulation, particle method, Vlasov equation, remapping, shape function, compact support

\section{Introduction}

The particle-in-cell (PIC) method [1, 2] is ubiquitous for simulating the dynamics of plasmas. A strength and weakness of the PIC method is the sparse representation of the particle distribution function in phase space. While this enables kinetic simulations in full six dimensional phase space, the method is also plagued by numerical noise. The desire to reduce the inherent noise in PIC simulations, combined with the increasing computational power available, has prompted renewed interest in grid based methods for solving

\footnotetext{
${ }^{*}$ Corresponding author.

Email address: larson6@llnl.gov (David J. Larson)
} 
the Vlasov equation, including various semi-Lagrangian schemes $[3,4,5]$, finite-element [6], and finite-volume based approaches [7].

While progress has been made using grid-based methods, the fundamental challenge of storing a six dimensional mesh remains a formidable hurdle. An alternative Lagrangian approach was initiated by Hewett [8] in which particles with finite extent in space and velocity are used to represent the distribution function. Initially the approach treated free expansion of the particles and used a heuristic merging technique. A subsequent extension [9] introduced refined merging techniques but only allowed for particle expansion and contraction along the coordinate axes, a fairly severe restriction. This approach was further developed by Lapenta and coworkers in $[10,11]$ by linearizing the forces and deriving equations to evolve the terms describing the particle envelope, including the covariance term, in one spatial and one velocity dimension. Alard and Colombi [12] extended this approach by allowing forces to vary quadratically over the particle envelope, using a tree-code for calculating the electric field, and utilizing the Richardson-Lucy algorithm [13] to remap the particles. Recent work $[14,15]$, again restricting the forces to linear variation over the particle envelope, uses a matrix to describe the particle extent and a finite-difference approximation to the Jacobian in order to evolve the particles.

The shape function kinetics (SFK) method reported here was inspired by the finite mass method (FMM) [16] developed in a series of papers by Yserentant and co-workers $[17,18,19,20,21]$ for grid-free fluid dynamics. The FMM has a solid mathematical foundation; it exactly conserves mass, momentum, energy, and angular momentum. For flows in a given velocity field or driven solely by external forces, the method has second-order spatial convergence [21]. However, fourth-order convergence has been observed when the particles interact via the laws of thermodynamics and are initialized with a fourth-order approximation of the initial velocity field [16].

We extend the FMM particle description to phase space and demonstrate the equivalence of the covariance matrix to the shape matrices describing the particle extent. Unlike the FMM and the other shape-function based methods cited above, equations for the time evolution of the shape matrix are not used to evolve the particle shape in time. Instead, the quadrature nodes associated with the shape function (Gaussians or cubic splines) are treated as PIC particles and the shape matrix is reconstructed from the covariance matrix calculated after the node positions and velocities are updated. This has the advantage of simplicity and obviates the need for storing three separate 
matrices per particle. The method is second-order in space, due to the use of Gaussian or cubic-spline basis functions, and time, due to the predictorcorrector time integration.

The paper is organized as follows: In Section 2 we describe the basic particle shape and the relationship between the various matrices. Section 3 describes the operations performed during each time step along with details concerning cubic splines and the two remapping procedures we investigated. Results of a number of test problems are then presented in Section 4, followed by a brief discussion concerning the addition of collisions in Section 5, and our conclusions.

\section{The Particle Model}

In the finite mass method [16] the macro particles are represented by differentiable shape functions of compact support. The shape function $\psi$ describes the distribution of mass within the particle envelope and takes only positive values. In order to demonstrate how the various matrices are related, we will use the exact expressions afforded by Gaussian shape functions in this section, despite the fact that they lack compact support. In position space $(\vec{x})$, the extent of particle $k$ is given by:

$$
\psi_{k}(\vec{x})=\frac{1}{(2 \pi)^{d_{x} / 2} \operatorname{Det}\left[\mathbf{H}_{\mathrm{x} k}\right]} \exp \left[-\frac{\vec{\xi}_{\mathrm{x} k} \cdot \vec{\xi}_{\mathrm{x} k}}{2}\right]
$$

where $d_{x}$ is the number of dimensions in physical space, $\mathbf{H}_{\mathrm{x} k}$ is the $d_{x} \times d_{x}$ spatial deformation matrix, and $\vec{\xi}_{\mathrm{x} k}$ is the scaled internal particle coordinate, defined by:

$$
\vec{\xi}_{\mathrm{x} k}=\mathbf{H}_{\mathrm{x} k}^{-1}\left(\vec{x}-\vec{x}_{k}\right)
$$

where $\vec{x}_{k}$ is the location of the particle's center of mass. The mass density is given by:

$$
\rho(\vec{x})=m \sum_{k=1}^{N_{p}} N_{k} \psi_{k}(\vec{x})
$$

where $m$ is the mass of a real particle (electron or ion), $N_{k}$ is the number of real particles (numerical weight) associated with the $k^{\text {th }}$ macro particle $\psi_{k}$, and $N_{p}$ is the total number of macro particles.

The internal velocity field $\vec{u}_{k}$ of the $k^{\text {th }}$ macro particle is expressed as:

$$
\vec{u}_{k}(\vec{x})=\vec{v}_{k}+\dot{\mathbf{H}}_{\mathrm{x} k} \cdot \vec{\xi}_{\mathrm{x} k}
$$


where $\vec{v}_{k}$ is the velocity of the particle center and $\dot{\mathbf{H}}_{\mathrm{x} k}$ is the time derivative of the $k^{\text {th }}$ particle's spatial shape matrix $\mathbf{H}_{\mathbf{x}}$. This shows that the internal velocity is restricted to a linear function of the spatial coordinates over the particle envelope.

In order to generalize these expressions to phase space, the particle description is extended to include velocity space $(\vec{v})$ :

$$
\psi_{k}(\vec{x}, \vec{v})=\frac{1}{(2 \pi)^{D / 2} \operatorname{Det}\left[\Sigma_{k}\right]^{1 / 2}} \exp \left[-\frac{\vec{\xi}_{\mathrm{x} k} \cdot \vec{\xi}_{\mathrm{x} k}}{2}\right] \exp \left[-\frac{\vec{\xi}_{\mathrm{v} k} \cdot \vec{\xi}_{\mathrm{v} k}}{2}\right]
$$

where $D=d_{x}+d_{v}$ is the total dimensionality of both physical $\left(d_{x}\right)$ and velocity $\left(d_{v}\right)$ space,

$$
\vec{\xi}_{\mathrm{v} k}=\mathbf{H}_{\mathrm{v} k}^{-1}\left[\vec{v}-\vec{u}_{k}(\vec{x})\right]
$$

is the scaled internal velocity coordinate of the $k^{\text {th }}$ particle, $\mathbf{H}_{\mathrm{v} k}$ is a $d_{v} \times d_{v}$ matrix describing the thermal velocity spread of the particle relative to its internal directed velocity, and the $D \times D$ covariance matrix $\left(\Sigma_{k}\right)$ is:

$$
\Sigma_{k}=\sum_{i} \sum_{j}\left(z_{i}-\mu_{i}\right)\left(z_{j}-\mu_{j}\right)^{T} .
$$

The indices in Eqn. (7) run over both spatial and velocity coordinates with $z_{i}$ designating the $i^{\text {th }}$ component of a general coordinate vector $\vec{z}$ and $\vec{\mu}$ the vector of generalized centroid locations. The phase space mass density is then given by:

$$
\rho(\vec{x}, \vec{v})=m \sum_{k=1}^{N_{p}} N_{k} \psi_{k}(\vec{x}, \vec{v}) .
$$

An illustration of the relationship between the various quantities for 1D phase space $\left(d_{x}=d_{v}=1\right)$ is given in Fig. 1. Figure 1a depicts an initially stationary Gaussian particle at $t=0$ with $\mathbf{H}_{\mathrm{x}}(0)=\mathbf{H}_{\mathrm{v}}(0)=1$ and $\dot{\mathbf{H}}_{\mathrm{x}}(0)=$ 0 . (Note that the deformation matrices reduce to single numbers in 1D phase space.) In the absence of external forces, the time evolution of a Gaussian spatial distribution and a Maxwellian velocity distribution can be described 
exactly by analytic expressions, as given by Hewett [9]:

$$
\begin{aligned}
& \mathbf{H}_{\mathrm{x}}(t)=\sqrt{\mathbf{H}_{\mathrm{x}}^{2}+\mathbf{H}_{\mathrm{v}}^{2} t^{2}} \\
& \dot{\mathbf{H}}_{\mathrm{x}}(t)=\frac{\mathbf{H}_{\mathrm{v}}^{2} t}{\sqrt{\mathbf{H}_{\mathrm{x}}^{2}+\mathbf{H}_{\mathrm{v}}^{2} t^{2}}} \\
& \mathbf{H}_{\mathrm{v}}(t)=\sqrt{\frac{\mathbf{H}_{\mathrm{x}}^{2} \mathbf{H}_{\mathrm{v}}^{2}}{\mathbf{H}_{\mathrm{x}}^{2}+\mathbf{H}_{\mathrm{v}}^{2} t^{2}}}
\end{aligned}
$$

where it is understood that the shape matrices on the right hand sides of the equations are evaluated at $t=0$. Figure $1 \mathrm{~b}$ shows the particle at $t=0.5$; note that energy is exactly conserved: $\mathbf{H}_{\mathrm{v}}^{2}(0.5)+\dot{\mathbf{H}}_{\mathrm{x}}^{2}(0.5)=\mathbf{H}_{\mathrm{v}}^{2}(0)=1$.

Integration over the particle shape function as given in Eqn. (5) reveals the following relation between the covariance matrix $\Sigma$ and the matrices used to define the particle extent in phase space:

$$
\Sigma=\left(\begin{array}{cc}
\mathbf{H}_{\mathrm{x}} \mathbf{H}_{\mathrm{x}} & \mathbf{H}_{\mathrm{x}} \dot{\mathbf{H}}_{\mathrm{x}} \\
\dot{\mathbf{H}}_{\mathrm{x}} \mathbf{H}_{\mathrm{x}} & \mathbf{H}_{\mathrm{v}} \mathbf{H}_{\mathrm{v}}+\dot{\mathbf{H}}_{\mathrm{x}} \dot{\mathbf{H}}_{\mathrm{x}}
\end{array}\right)=\mathbf{H H}
$$

This expression also defines the $D \times D$ deformation matrix $\mathbf{H}$ that specifies the particle extent in phase space. This relationship, with suitable normalization, also holds for shape functions constructed from the tensor product of cubic B-splines and suggests the method used to evolve the particle envelope described below. For completeness, the covariance matrices of the example particles in Fig. 1 are:

$$
\Sigma(0)=\left(\begin{array}{ll}
1 & 0 \\
0 & 1
\end{array}\right) \quad \text { and } \quad \Sigma(0.5)=\left(\begin{array}{cc}
1.25 & 0.5 \\
0.5 & 1.0
\end{array}\right)
$$

\section{Algorithm}

\subsection{Order of calculations}

In order to represent the phase space density at a given time, the particle positions and velocities must both be known at that time. This disqualifies the standard leap-frog scheme used in many PIC codes because it staggers positions and velocities in time. We use the second-order predictor-corrector algorithm instead, as in [12] and [14].

The following steps are used to advance the particles and fields in time: 


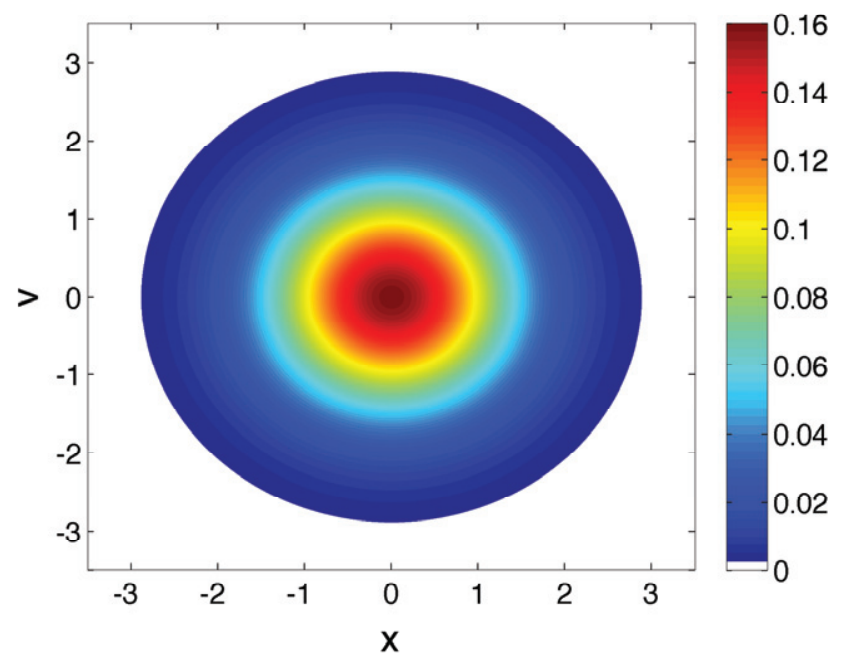

(a) $\mathrm{t}=0$

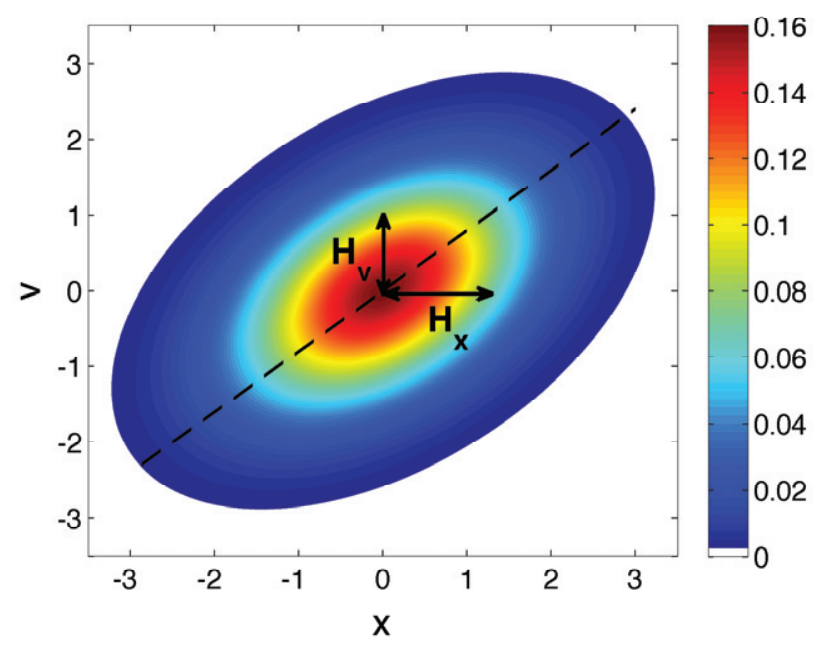

(b) $\mathrm{t}=0.5$

Figure 1: (a) Initial particle extent in phase space at $t=0$ with $\mathbf{H}_{\mathrm{x}}(0)=1, \dot{\mathbf{H}}_{\mathrm{x}}(0)=0$, and $\mathbf{H}_{\mathrm{v}}(0)=1$. (b) Free evolution of the particle in (a) evaluated at $t=0.5$ with $\mathbf{H}_{\mathrm{x}}(0.5)=1.11803, \dot{\mathbf{H}}_{\mathrm{x}}(0.5)=0.447214$, and $\mathbf{H}_{\mathrm{v}}(0.5)=0.894427$. The Gaussian particles have been truncated at 3 standard deviations in each dimension. The dashed line in (b) indicates the instantaneous velocity of the particle symmetry axis. The slope is related to the time rate of change of the particle length scale: $2 \dot{\mathbf{H}}_{\mathrm{x}} / \mathbf{H}_{\mathrm{x}}=0.8$. 
1. Initialize $N_{p}$ macro particles on a regular mesh in phase space with weights $N_{k}$ using cubic B-splines (see Sec. 3.2). The normalized thirdorder B-spline used in this work is:

$$
\begin{array}{ll}
\psi(\xi)=\frac{1}{6}\left[4-3|\xi|^{2}(2-|\xi|)\right] & ; \quad 0 \leq|\xi| \leq 1 \\
\psi(\xi)=\frac{1}{6}(2-|\xi|)^{3} & ; \quad 1 \leq|\xi| \leq 2 .
\end{array}
$$

This spline has the following properties:

$$
\int_{-2}^{2} \psi(\xi) d \xi=1 \quad ; \quad \int_{-2}^{2} \xi \psi(\xi) d \xi=0 \quad ; \quad \int_{-2}^{2} 3 \xi^{2} \psi(\xi) d \xi=1 .
$$

The extension to $d$ higher dimensions is the tensor product:

$$
\psi(\vec{\xi})=\prod_{i=1}^{d} \psi\left(\xi_{i}\right)
$$

2. Construct a set of point particles at the quadrature nodes of the macro particle shape function with appropriate locations, $\vec{x}_{i}$, velocities, $\vec{v}_{i}$, and weights $\alpha_{i}$ (see Sec. 3.3).

3. Following the second-order predictor-corrector scheme, advance the point particles half a time step from their position at the current time level $n$ to the $t+\frac{\Delta t}{2}$ time level:

$$
\vec{x}_{i}^{n+1 / 2}=\vec{x}_{i}^{n}+\frac{\Delta t}{2} \vec{v}_{i}^{n} .
$$

4. Accumulate the charge density $\rho^{n+1 / 2}$ on the spatial mesh using linear interpolation, where a point particle gives a fraction of its charge to each of the neighboring grid points in inverse proportion to the separation distance. Each macro particle carries a total charge $q_{k}=N_{k} q$, where $q= \pm e$ is the fundamental charge. A macro particle's charge has the same phase space distribution as its mass.

5. Calculate the electric field $E$ by solving Poisson's equation for the electrostatic potential $\phi$ using standard second-order finite difference methods:

$$
\begin{aligned}
\nabla^{2} \phi^{n+1 / 2} & =-4 \pi \rho^{n+1 / 2} \\
E^{n+1 / 2} & =-\nabla \phi^{n+1 / 2}
\end{aligned}
$$




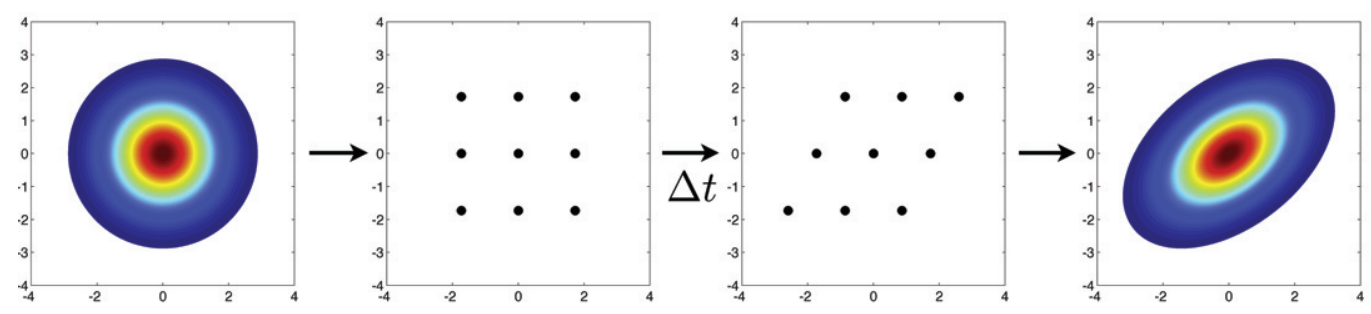

Figure 2: The evolution of a freely expanding macro particle is accomplished by advancing the underlying set of weighted point particles and subsequently reconstructing the particle envelope.

6. Update the point particle velocities by a full time step $\Delta t$ :

$$
\vec{v}_{i}^{n+1}=\vec{v}_{i}^{n}+\Delta t\left(\frac{q}{m}\right) E^{n+1 / 2} .
$$

7. Advance the point particle positions the remaining half step in time to $t+\Delta t:$

$$
\vec{x}_{i}^{n+1}=\vec{x}_{i}^{n+1 / 2}+\frac{\Delta t}{2} \vec{v}_{i}^{n+1} .
$$

8. For each particle $k$, construct the covariance matrix from the associated point particles. Calculate the new deformation matrix $\mathbf{H}_{k}=\sqrt{\boldsymbol{\Sigma}_{k}}$.

9. If a particle remap is required, return to step (1); otherwise, return to step (2).

The process of generating weighted point particles, advancing them in time, and constructing a new macro particle envelope from the updated set is illustrated in Fig. 2.

\subsection{Initialization and remapping}

Step (1) is accomplished using the Lucy-Richardson algorithm, as discussed in [12] and illustrated in Fig. 3. The initial values of the phase space density $f_{0}(x, v)$ are defined on a uniform $N_{x} \times N_{v}$ mesh with spacing $(\Delta x, \Delta v)$ in $(x, v)$. The $N_{p}=N_{x} \times N_{v}$ macro particles are initialized at the mesh points with deformation matrices initialized to:

$$
\mathbf{H}_{0}=\left(\begin{array}{cc}
\Delta x & 0 \\
0 & \Delta v
\end{array}\right) .
$$

The particle weights are set by the value of the phase space density at the mesh points, so the $i^{\text {th }}$ particle located at point $\left(x_{i}, v_{i}\right)$ will be assigned a 

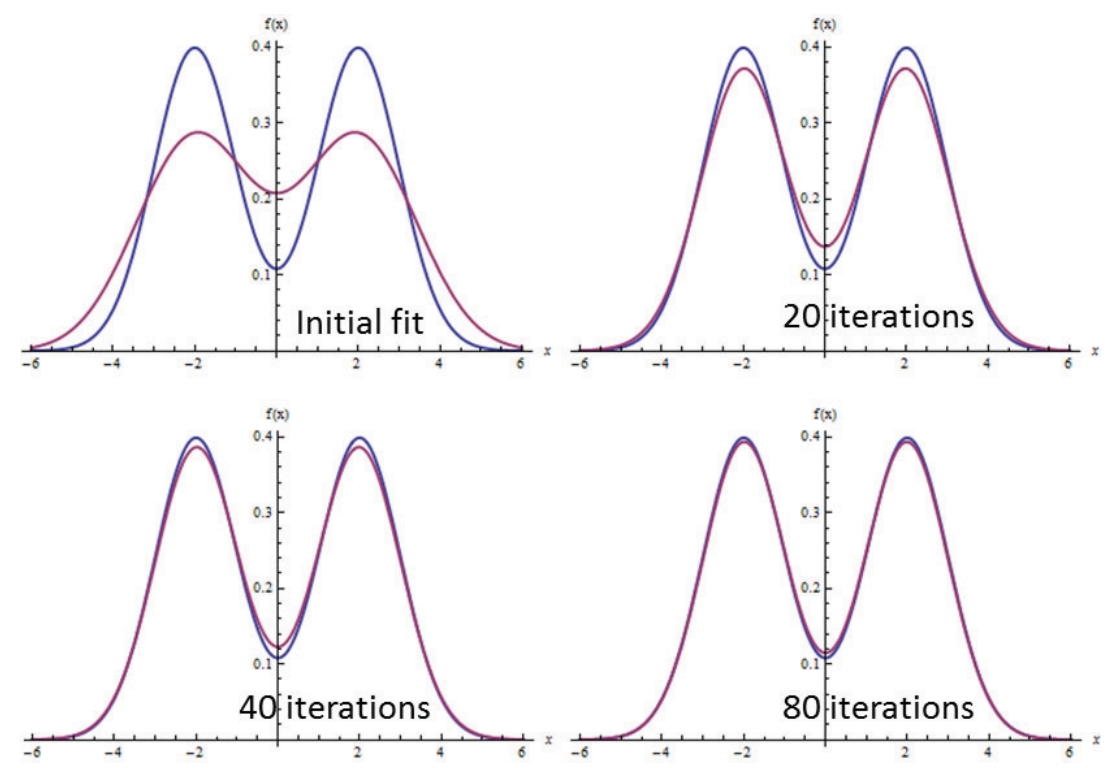

Figure 3: The Richardson-Lucy algorithm applied to fitting a set of nine equally-spaced Gaussian basis functions to a given curve.

weight $N_{i}=f_{0}\left(x_{i}, v_{i}\right) \Delta x \Delta v$. This yields an initial approximation $\widetilde{f}_{0}$ to the true phase space density $f_{0}$ :

$$
\tilde{f}_{0}(x, v)=\sum_{k=1}^{N_{p}} \frac{N_{k}}{\operatorname{Det}\left[\mathbf{H}_{k}\right]} \psi\left(\vec{\xi}_{k}\right)=\sum_{i=1}^{N_{x} N_{v}} f_{0}\left(x_{i}, v_{i}\right) \psi\left(\vec{\xi}_{i}\right)
$$

since all the deformation matrices $\mathbf{H}_{k}=\mathbf{H}_{0}$ at initialization. The internal particle coordinate $\vec{\xi}_{k}=\vec{\xi}_{i}=\mathbf{H}_{0}^{-1}\left(\vec{z}-\vec{\mu}_{i}\right)$ where $\vec{z}=(x, v)$ is the generalized coordinate vector and $\vec{\mu}_{i}=\left(x_{i}, v_{i}\right)$ is the grid location of particle $i$ 's centroid. The Richardson-Lucy algorithm, illustrated in Fig. 3, iteratively adjusts the particle weights in order to produce the best fit $\widetilde{f}_{m}\left(x_{i}, v_{i}\right) \rightarrow f_{0}\left(x_{i}, v_{i}\right)$ while ensuring that $\tilde{f}_{m}$ remains positive. The iteration at mesh point $i$ is:

$$
\begin{aligned}
\delta_{i}^{m} & =\frac{f_{0}\left(x_{i}, v_{i}\right)}{\widetilde{f}_{m}\left(x_{i}, v_{i}\right)}-1 \\
N_{i}^{m+1} & =N_{i}^{m} \sum_{j}\left(\frac{1+\delta_{j}^{m}}{\operatorname{Det}\left[\mathbf{H}_{0}\right]}\right) \psi\left(\frac{x_{i}-x_{j}}{\Delta x}, \frac{v_{i}-v_{j}}{\Delta v}\right) .
\end{aligned}
$$

Note that this iteration does not change the deformation matrices $\mathbf{H}$, which remain equal to $\mathbf{H}_{0}$. 
The accuracy of the simulation will degrade over time as the particles distort due to the applied forces and their internal velocity spread. It is necessary to periodically remap the macro particles onto a fresh undistorted basis set. This is done by first accumulating the phase space density from the point particles at the current time step $n, f_{n}\left(x_{i}, v_{i}\right)$, on the same uniform mesh in phase space as above. The analysis in [22], for a purely PIC-based approach, demonstrates that at least third-order interpolation is required in the remapping step to achieve second-order for the overall method. Instead of using higher-order interpolation, we employ linear interpolation and a higher-order quadrature rule when accumulating the phase space density in the remapping step. The accumulated $f_{n}$ then becomes the initial $f_{0}$ used as a reference in the Lucy-Richardson iteration.

Alard and Colombi [12] present a thorough analysis of the Lucy-Richardson iteration and point out that it is susceptible to aliasing effects. We typically use 5-7 iterations in order to achieve a reasonable balance between minimizing diffusion and avoiding aliasing. Experience has shown that using either the 2-point or 3-point quadrature rule (see Table 1) when accumulating the phase space density prior to remapping leads to an increase in aliasing effects and a corresponding loss of fidelity. The 5-point quadrature rule, which is exact for fifth-order polynomials $[16,23]$, is used in the remap procedure for all of the results presented below.

We have also investigated particle remapping using a combination of a tree algorithm [24] and the expectation-maximization (EM) algorithm [25]. The EM algorithm fits a selected number of Gaussian basis functions to a set of scattered data points. After the point particles are sorted into uniformly sized boxes by the tree code, the center of mass for the particles in a given box is calculated. The center of mass locations then become the initial centroids of a new Gaussian basis set calculated using the mixture model. Note that since the Gaussian mixture model adjusts both the particle envelope and the numerical weight, non-uniform particle envelopes $(\mathbf{H})$ are a natural outcome. This works well, as shown in Fig. 4, and has the advantage that it captures small scale structure. Unfortunately, it requires an excessive amount of time to compute, in our naïve implementation, because the standard mixture model scales like $N_{\mathrm{qp}}^{2}$, where $N_{\mathrm{qp}}$ is the total number of quadrature points. However, there are known methods for speeding up mixture models [26] which might well make the approach tractable. 

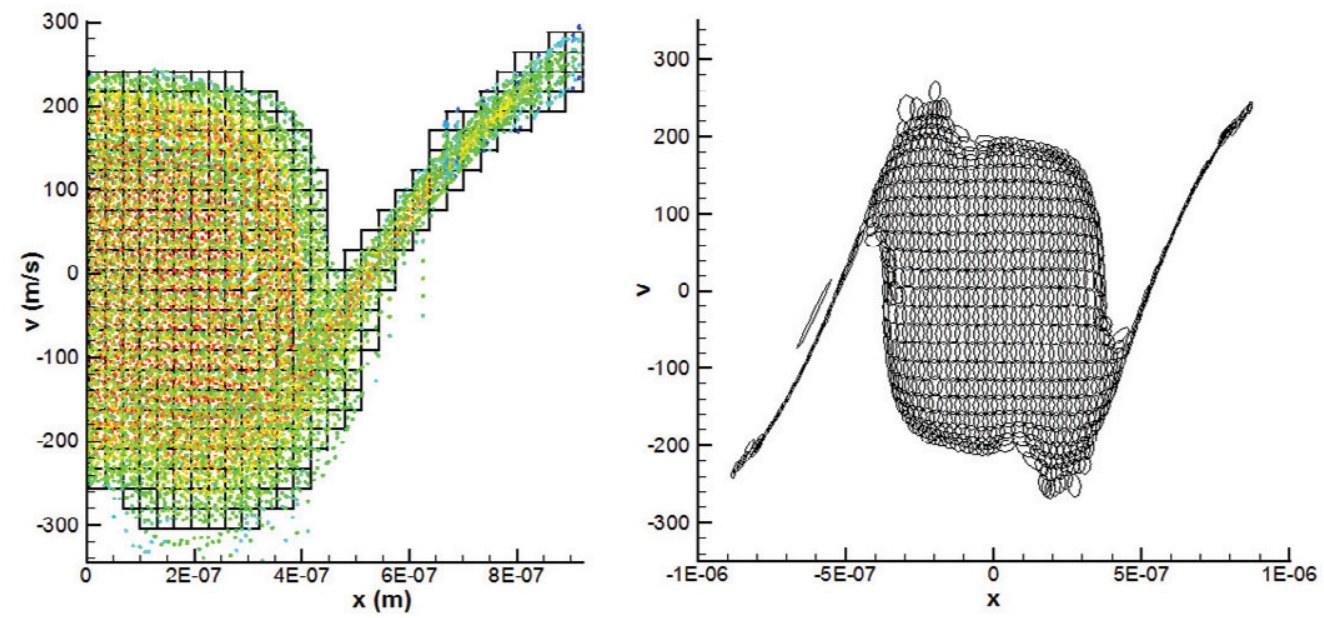

Figure 4: (a) A point particle set, with color denoting the numerical weight, sorted into uniform boxes using a tree code. (b) The resulting set of particle envelopes produced by the Gaussian mixture EM algorithm. Note that this procedure generates non-uniform particle envelopes and resolves the developing fine structure.

\subsection{Generating point particles at the quadrature nodes}

The shape functions represent the distribution of mass lying within the particle envelope and have strictly positive values. This should be reflected in the quadrature rules employed. The usual Gauss-Hermite quadrature rules apply for Gaussian shape functions. For cubic B-splines, we follow Yserentant and co-workers $[16,21,23]$ and employ the tensor-product counterparts of the weighted quadrature rules given in Table 1 . The 2 and 3-point rules are derived via Gaussian quadrature.

For each macro particle $k$, point particles are generated at the quadrature nodes using the tensor product of the selected one-dimensional quadrature rule. The locations are given by:

$$
\left(\begin{array}{c}
\vec{x}_{i} \\
\vec{v}_{i}
\end{array}\right)=\left(\begin{array}{l}
\vec{x}_{k} \\
\vec{v}_{k}
\end{array}\right)+\mathbf{H}_{k}\left(\begin{array}{l}
\vec{y}_{x} \\
\vec{y}_{v}
\end{array}\right)
$$

where $\vec{y}$ is the vector of quadrature nodes. Each point particle $i$ belonging to particle $k$ carries a mass $m_{i}=m N_{k} \alpha_{i}$ with the quadrature weight $\alpha_{i}>0$ and equal to the appropriate product of the quadrature rule weights. For example, in 1D phase space using the 3-point rule, the point particle with nodes $y_{x}=-\frac{3}{\sqrt{10}}$ and $y_{v}=0$ will have $\alpha_{i}=\frac{85}{729}$. 
Table 1: One-dimensional quadrature rules for cubic B-splines.

\begin{tabular}{|c|c|c|c|c|c|}
\hline \multicolumn{2}{|c|}{ (a) 2 Points } & \multicolumn{2}{|c|}{ (b) 3 Points } & \multicolumn{2}{|c|}{ (c) 5 Points } \\
\hline Nodes & Weights & Nodes & Weights & Nodes & Weights \\
\hline$-\frac{1}{\sqrt{3}}$ & $\frac{1}{2}$ & $-\frac{3}{\sqrt{10}}$ & $\frac{5}{27}$ & $-\frac{4}{3}$ & $\frac{41}{1280}$ \\
\hline$\frac{1}{\sqrt{3}}$ & $\frac{1}{2}$ & 0 & $\frac{17}{27}$ & $-\frac{2}{3}$ & $\frac{316}{1280}$ \\
\hline & & $\frac{3}{\sqrt{10}}$ & $\frac{5}{27}$ & 0 & $\frac{566}{1280}$ \\
\hline & & & & $\frac{2}{3}$ & $\frac{316}{1280}$ \\
\hline & & & & $\frac{4}{3}$ & $\frac{41}{1280}$ \\
\hline
\end{tabular}

\subsection{Constructing the covariance matrix}

After the point particles are advanced in time, the covariance matrix for each macro particle $k$ is constructed. First, the center of mass position and centroid velocity are computed:

$$
\vec{x}_{k}^{n+1}=\frac{\sum_{i=1}^{N_{\mathrm{qp}}} m_{i} \vec{x}_{i}^{n+1}}{\sum_{i=1}^{N_{\mathrm{qp}}} m_{i}} ; \quad \vec{v}_{k}^{n+1}=\frac{\sum_{i=1}^{N_{\mathrm{qp}}} m_{i} \vec{v}_{i}^{n+1}}{\sum_{i=1}^{N_{\mathrm{qp}}} m_{i}}
$$

where $N_{\mathrm{qp}}$ is the total number of point particles per macro particle $k$. Then the covariance matrix is computed:

$$
\boldsymbol{\Sigma}_{k}^{n+1}=\frac{\sum_{i=1}^{N_{\mathrm{qp}}} m_{i} \sum_{j} \sum_{m}\left(\vec{z}_{j}^{n+1}-\vec{\mu}_{k j}^{n+1}\right)\left(\vec{z}_{m}^{n+1}-\vec{\mu}_{k m}^{n+1}\right)^{T}}{\sum_{i=1}^{N_{\mathrm{qp}}} m_{i}}
$$

where $\vec{z}=\left(\vec{x}_{i}, \vec{v}_{i}\right)$ and $\vec{\mu}_{k}=\left(\vec{x}_{k}, \vec{v}_{k}\right)$. The updated deformation matrix is then equal to the square root of the symmetric matrix $\Sigma_{k}^{n+1}$ per Eqn. (12):

$$
\mathbf{H}_{k}^{n+1}=\sqrt{\Sigma_{k}^{n+1}}
$$




\section{Test Problems}

A calculation to examine relative noise levels and three test problems are considered in $(x, v)$ space with periodic boundaries in the $x$ dimension. Results for two Landau damping problems are presented along with those from a two stream instability problem. All calculations were run on a uniform mesh with $-2 \pi \leq x \leq 2 \pi$ and $-2 \pi \leq v \leq 2 \pi$. We set $m_{\mathrm{e}}=1, q_{\mathrm{e}}=-1$, and follow the electron dynamics in the presence of a constant ion density $n_{\mathrm{i}}=1$.

\subsection{Quiescent Plasma}

We first consider the fluctuations in the electric field energy for quiescent plasma $\left(n_{\mathrm{e}}=1, T_{\mathrm{e}}=1\right)$ over 30 plasma periods. Results obtained using the present method with $512 \times 512$ particles, the 2-point quadrature rule $\left(N_{\mathrm{SFK}}=512 \times 512 \times 4=1,048,576\right)$, and a field solution calculated on a 64 cell mesh are plotted in Fig. 5 along with results from standard PIC calculations using linear weighting and equally weighted particles. The PIC

results follow the well-known $1 / \sqrt{N}$ scaling and would require many more particles in order to match the SFK result.

The SFK code and the PIC code are identical apart from the additional subroutines required for generating the quadrature points, calculating the covariance matrix, etc. The SFK run required approximately 56 seconds running in double precision on a $2.9 \mathrm{GHz} \mathrm{PC}$, while the PIC calculations required 18 seconds ( $N_{\mathrm{SFK}}$ particles), 43 seconds $\left(4 \times N_{\mathrm{SFK}}\right.$ particles), and 152 seconds $\left(16 \times N_{\text {SFK }}\right.$ particles $)$. The run times indicate that time per quadrature node for SFK is slightly more than three times the time per particle for standard PIC. The additional run time seems well worth the factor of $\sim 10^{12}$ reduction in energy fluctuations.

\subsection{Landau Damping}

For the two Landau damping problems, also considered in $[4,5,7,14]$, the initial distribution function is:

$$
f(x, v)=\frac{1}{\sqrt{2 \pi}} \exp \left(-\frac{v^{2}}{2}\right)[1+\alpha \cos (k x)]
$$

with $k=0.5$ and $\alpha=0.01$ for what we will label the linear weak Landau damping case and $\alpha=0.5$ for the nonlinear strong Landau damping case.

Figure 6 illustrates how the frequency of remapping affects the damping rate in the weak Landau damping case and how the noise in a standard PIC 


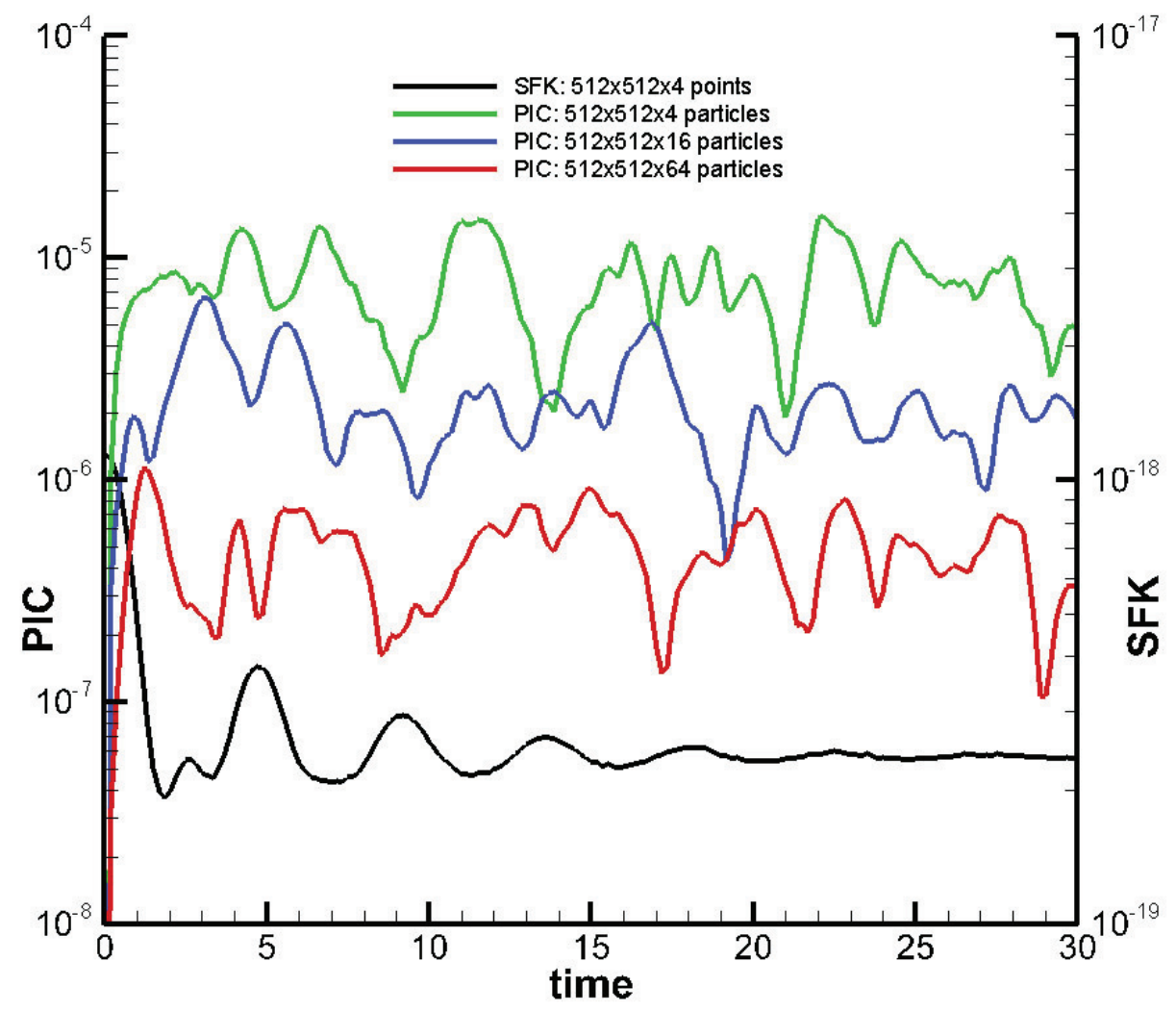

Figure 5: Fluctuations in electric field energy for quiescent plasma with $1 / \omega_{\mathrm{pe}}=1$. PIC results are plotted against the scale at left, SFK result against the right $(\Delta t=1 / 6$ for all calculations).

code disrupts the expected decay of the field energy. The calculations used $64 \times 64$ particles, the two-point quadrature rule, and a time step $\Delta t=1 / 8$. The mesh for the Poisson solution had 64 cells. The straightforward remapping procedure is mildly diffusive due to the interpolation used to accumulate the phase space density. This could be alleviated by employing adaptive mesh refinement (AMR) or using the tree code and Gaussian mixture model combination discussed above. The results demonstrate that even with 100 times as many degrees of freedom $(256 \times 256 \times 25$ PIC particles compared to $64 \times 64 \times 4$ quadrature point particles) the standard PIC approach can't 


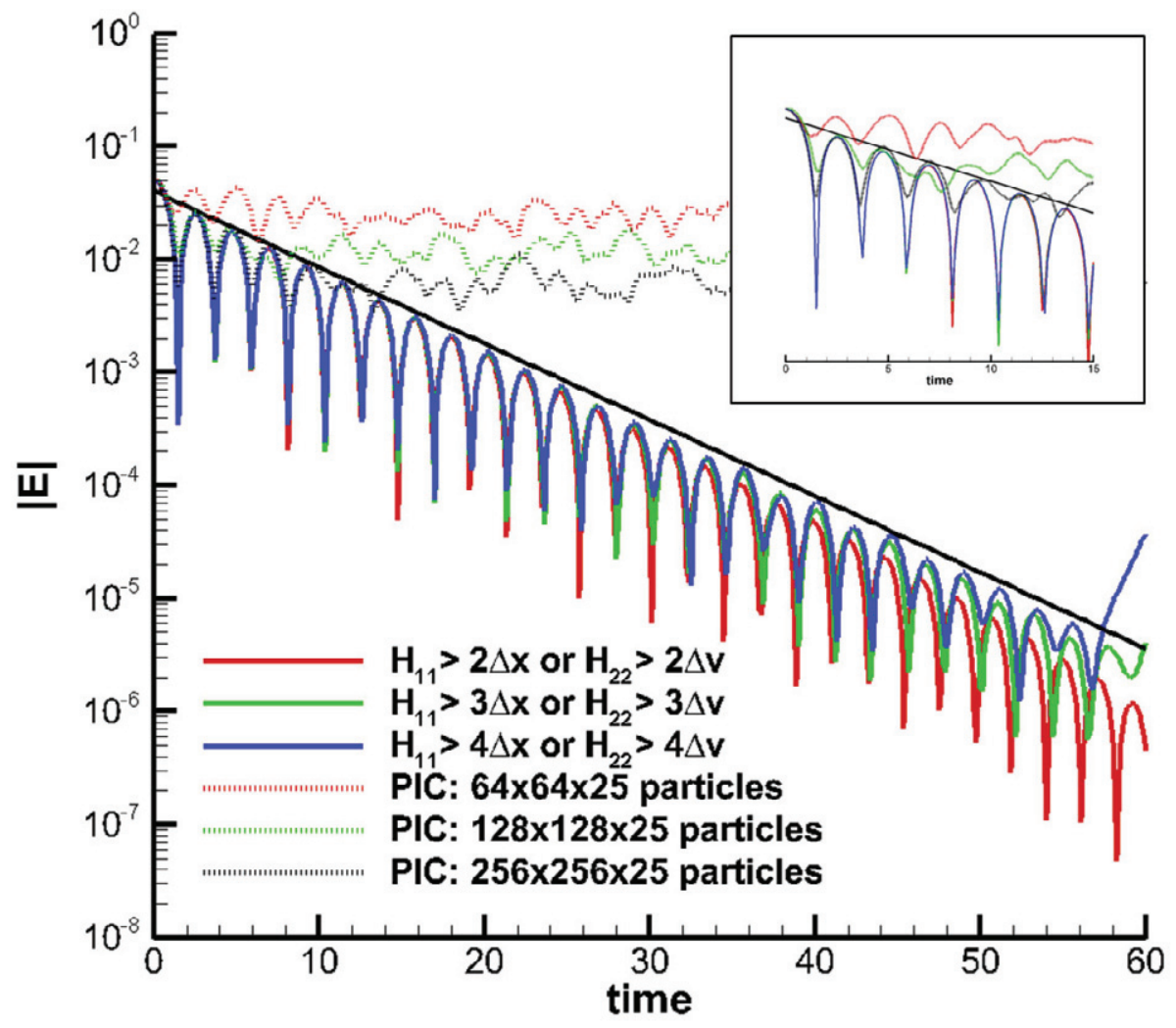

Figure 6: $L_{2}$ norm of the electric field energy plotted versus time in the weak Landau damping case. The curves correspond to results generated using different criteria to trigger remapping. A remap occurs approximately every 14, 23, and 31 time steps for the respective criteria $h>2 \Delta x, h>3 \Delta x$, and $h>4 \Delta x$. The black line corresponds to the theoretical damping rate $\gamma=-0.1553$. The increase in the blue curve at $t \approx 60$ is the beginning of a Poincare recurrence.

follow the decay of the field energy beyond $t \approx 10$, in agreement with the results obtained in [14]. All of the results presented below, unless otherwise specified, were produced using the criteria that a remap was performed if $H_{11} \geq 4 \Delta x$ or $H_{22} \geq 4 \Delta v$.

The Lagrangian nature of the method is illustrated in Fig. 7 where several early snapshots of the particle envelopes from the strong Landau damping case are plotted. The corresponding phase space plots are shown in Fig. 8 
using the results from a run with $256 \times 256$ particles, 128 cells for the electric field calculation, and the 2-point quadrature rule.

Results for the strong Landau case show little variation, apart from some indication of aliasing effects in the 2-point case late in time, with regard to the quadrature rule used, as shown in Fig 9. This is as expected because the 2-point rule is exact for third-order polynomials and the particle shape functions are accurate to second-order in space. As long as the particle scale length is less than the cell size used in the electric field solve, there is no benefit to utilizing higher-order quadrature rules to evolve the particles.

The run times for the three simulations reflect the advantage of using the minimum number of quadrature points: 82, 93, and 150 seconds for the 2 , 3 , and 5-point rules, respectively. The run time should scale like $N_{\mathrm{qp}}^{D}$, where $N_{\mathrm{qp}}$ is the number of points in the rule, thus the timings indicate a sizeable overhead due to remapping, diagnostics, etc. The use of the tensor product of one-dimensional quadrature rules can be problematic in higher dimensions due to the $N_{\mathrm{qp}}^{D}$ scaling [27], and we note that quadrature rules of degree 3 using only $2 D$ points [28] exist for Gaussian shape functions.

The evolution of the phase space density is shown in Fig. 10, demonstrating that the fine structure is accurately resolved. (These plots may be compared with Fig. 6 in [14] and Fig. 10 in [4].) The phase space density at the final time computed with different particle resolutions is plotted in Fig. 11. Both calculations used the 2-point quadrature rule and remapped when $H_{11} \geq 2 \Delta x$ or $H_{22} \geq 2 \Delta v$. Figure 10 and the plot on the left in Fig. 11 display results obtained using $256 \times 256$ particles and 128 cells in the Poisson solve. The calculation ran in 39.1 seconds. The results on the right side of Fig. 11 were obtained in 196 seconds using $512 \times 512$ particles and 256 cells in the Poisson solve.

\subsection{Two-Stream Instability}

The last example problem is the two-stream instability where the initial phase space density is given by:

$$
f(x, v)=\frac{v^{2}}{\sqrt{2 \pi}} \exp \left(-\frac{v^{2}}{2}\right)\left[1-\frac{1}{2} \cos \left(\frac{x}{2}\right)\right] .
$$

The effects of varying resolution can be seen in Fig. 12 where the $L_{2}$ norm of $f$ is plotted as a function of time. The results at all resolutions compare favorably with Fig. 7 in [4] where a much more complicated fouth-order 

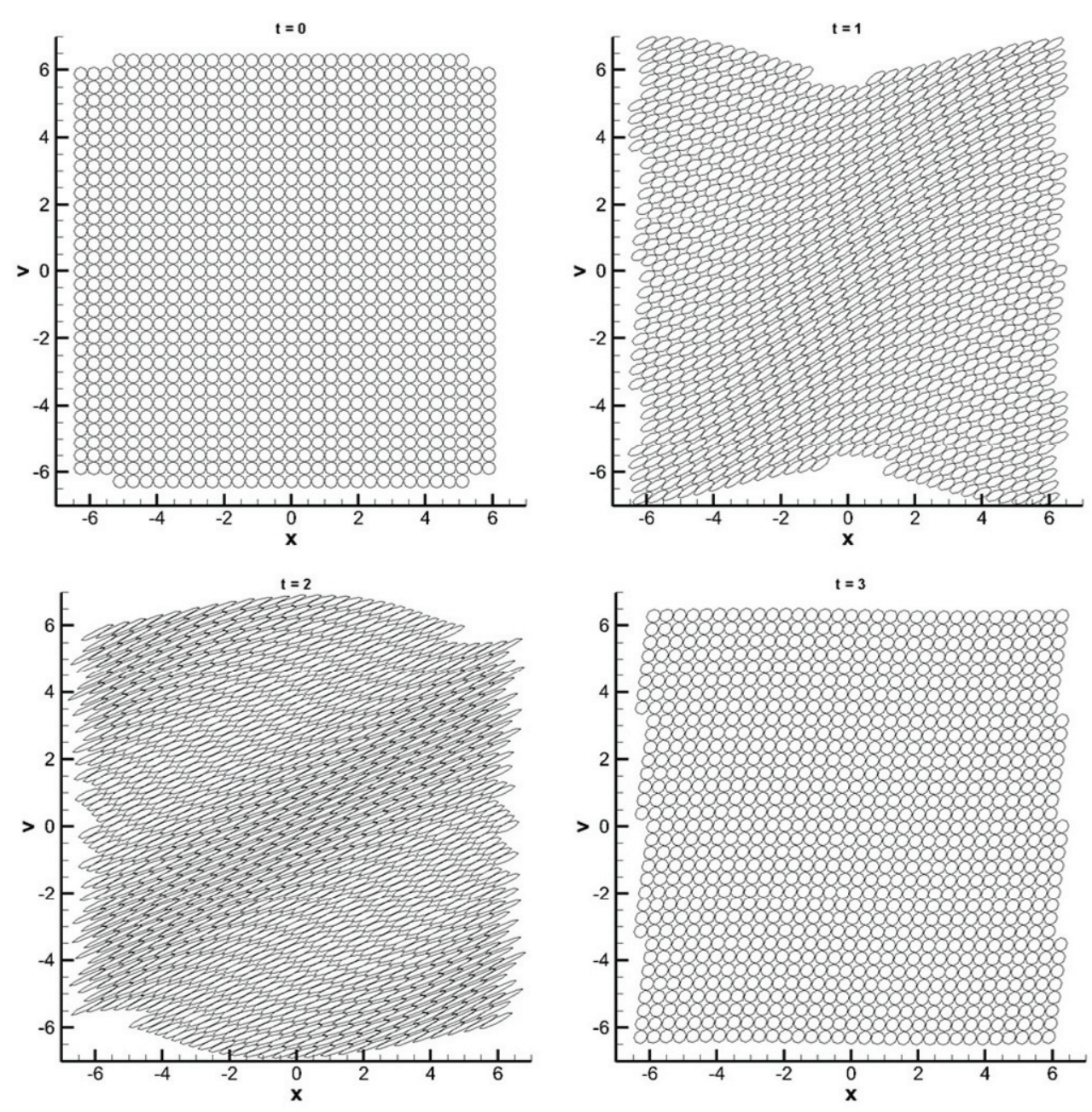

Figure 7: Particle envelopes for the strong Landau damping case. A small number of particles $(32 \times 32)$ were initialized in order to clearly display their Lagrangian evolution. The final plot at $t=3$ is one time step after a remapping. The outlines are drawn using $0.5 \mathbf{H}$ to avoid showing their true overlap.

scheme is used. As mentioned above, the particles were remapped when $H_{11} \geq 4 \Delta x$ or $H_{22} \geq 4 \Delta v$ and these remappings are clearly distinguishable in the plot. The majority of the error growth occurs due to the remapping. It is also clear that using the 5-point rule reduces the oscillations that occur just prior to a remap. The oscillations indicate that remapping should be done more often; as illustrated in Fig. 13, the amplitude drops substantially 

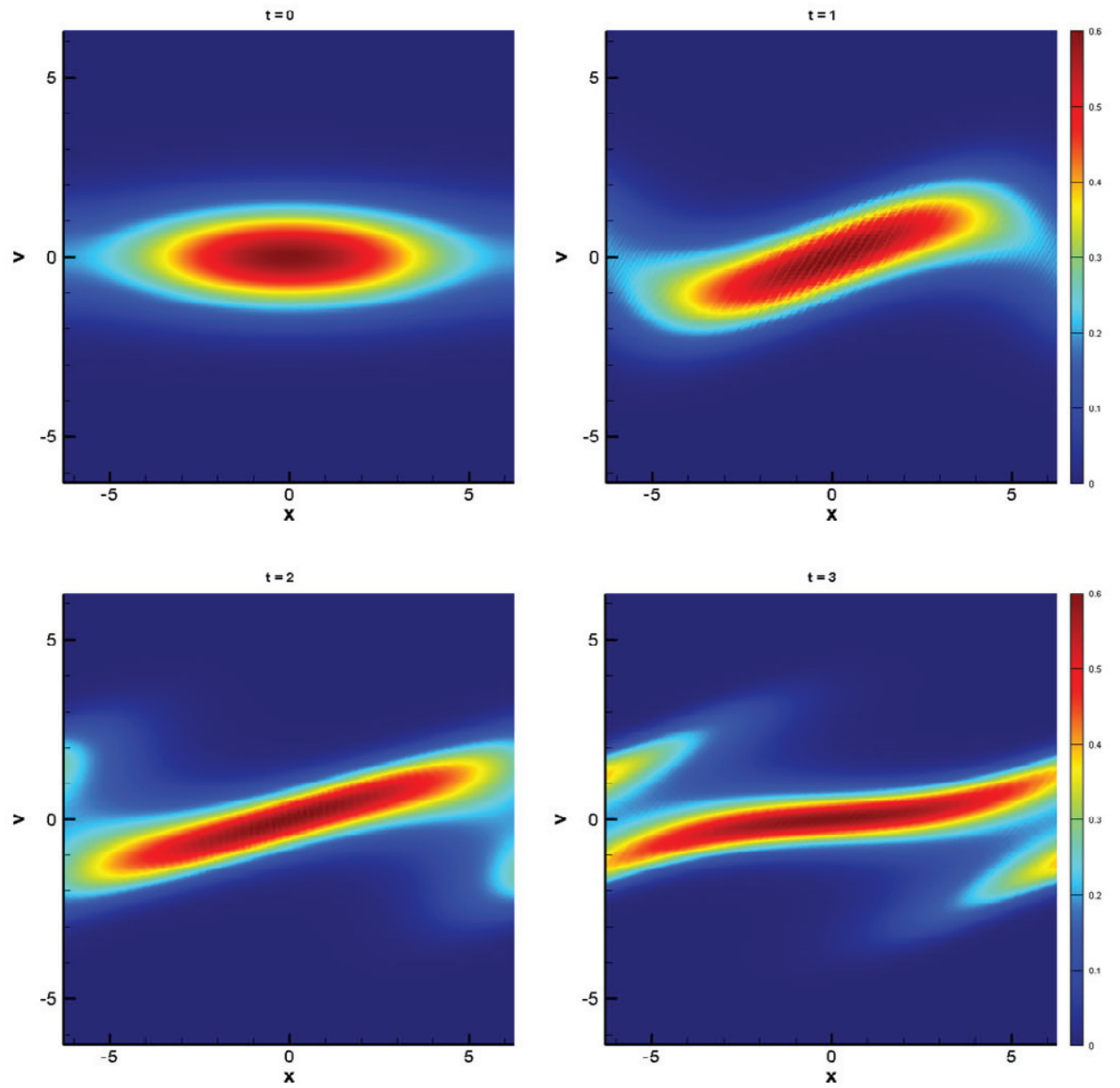

Figure 8: Phase space density for the strong Landau damping case using $256 \times 256$ particles at times corresponding to those shown in Fig. 7.

when this is the case. Run time increases when the remapping frequency increases and the error in the $L_{2}$ norm also increases slightly. The resulting (nearly indistinguishable) phase space plots at $t=45$ are shown in Fig. 14.

Finally, we demonstrate the convergence properties of the method. We keep the ratio of the initial particle mesh spacing to the Poisson solver mesh spacing constant, $r_{h}=h_{x} / \Delta x_{P}=1 / 2$. The relative error in the total energy is defined as

$$
e^{2 h}=\frac{\left|E^{2 h}-E^{h}\right|}{E^{h}}
$$




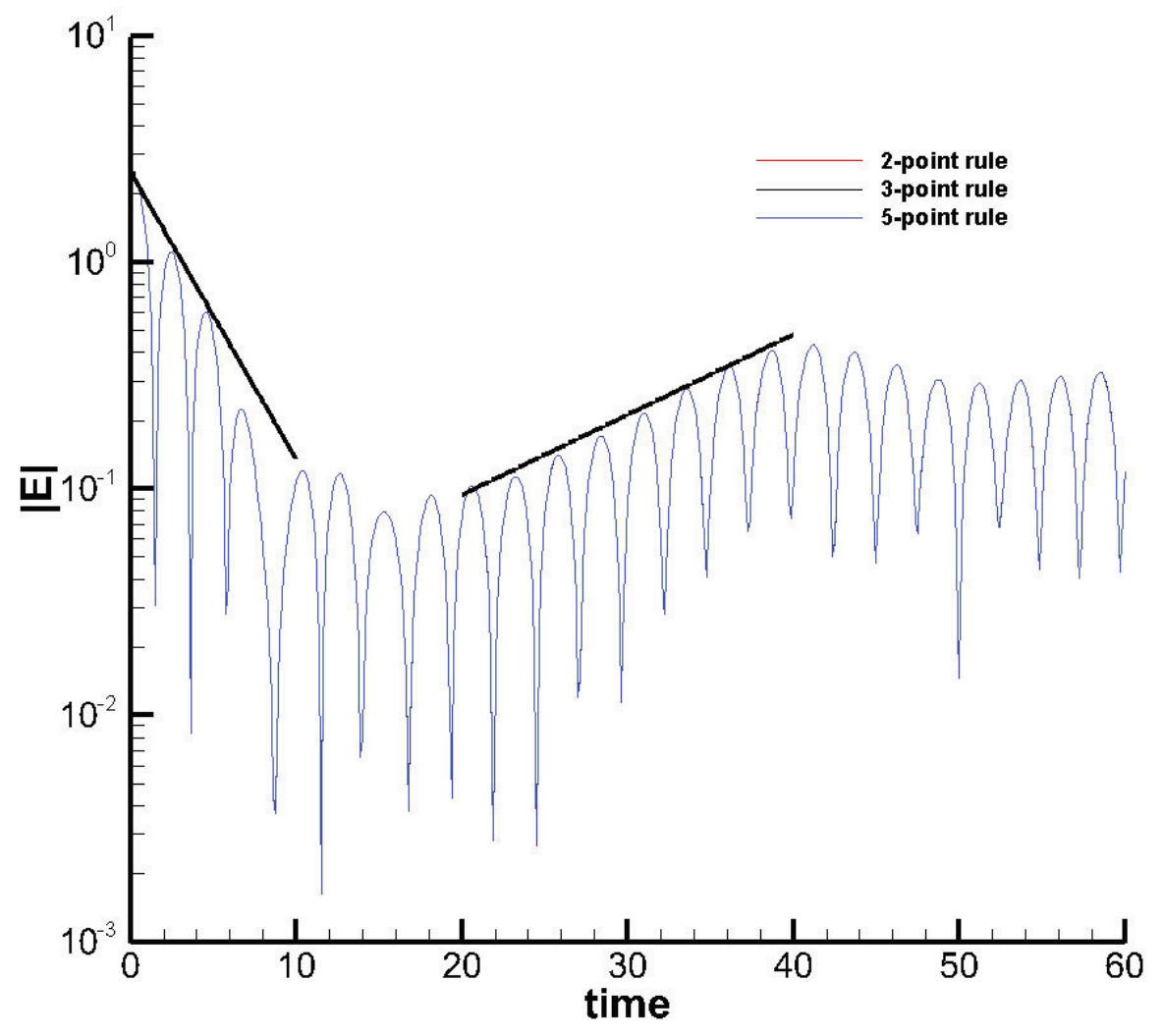

Figure 9: The $L_{2}$ norm of the electric field energy for the strong Landau damping case using different quadrature rules. The curves are indistinguishable. Also plotted are the initial damping rate $\gamma_{1}=-0.292$ and the growth rate $\gamma_{2}=0.0815$, for $20 \leq t \leq 30$, as computed by [4].

where $E^{h}$ is the sum of the electric field energy and the kinetic energy computed with initial phase-space discretization $\left(h_{x}, h_{v}\right)$ and time integration step $\Delta t . E^{2 h}$ is computed with $\left(2 h_{x}, 2 h_{v}\right)$ and $2 \Delta t$. The order of the method, $q$, is then given by

$$
q=\log _{2}\left(\frac{e^{2 h}}{e^{h}}\right) .
$$

As the resolution increases, the filamentary structure developing in phase space is captured with increasing fidelity, as shown in Fig. 15, which may 

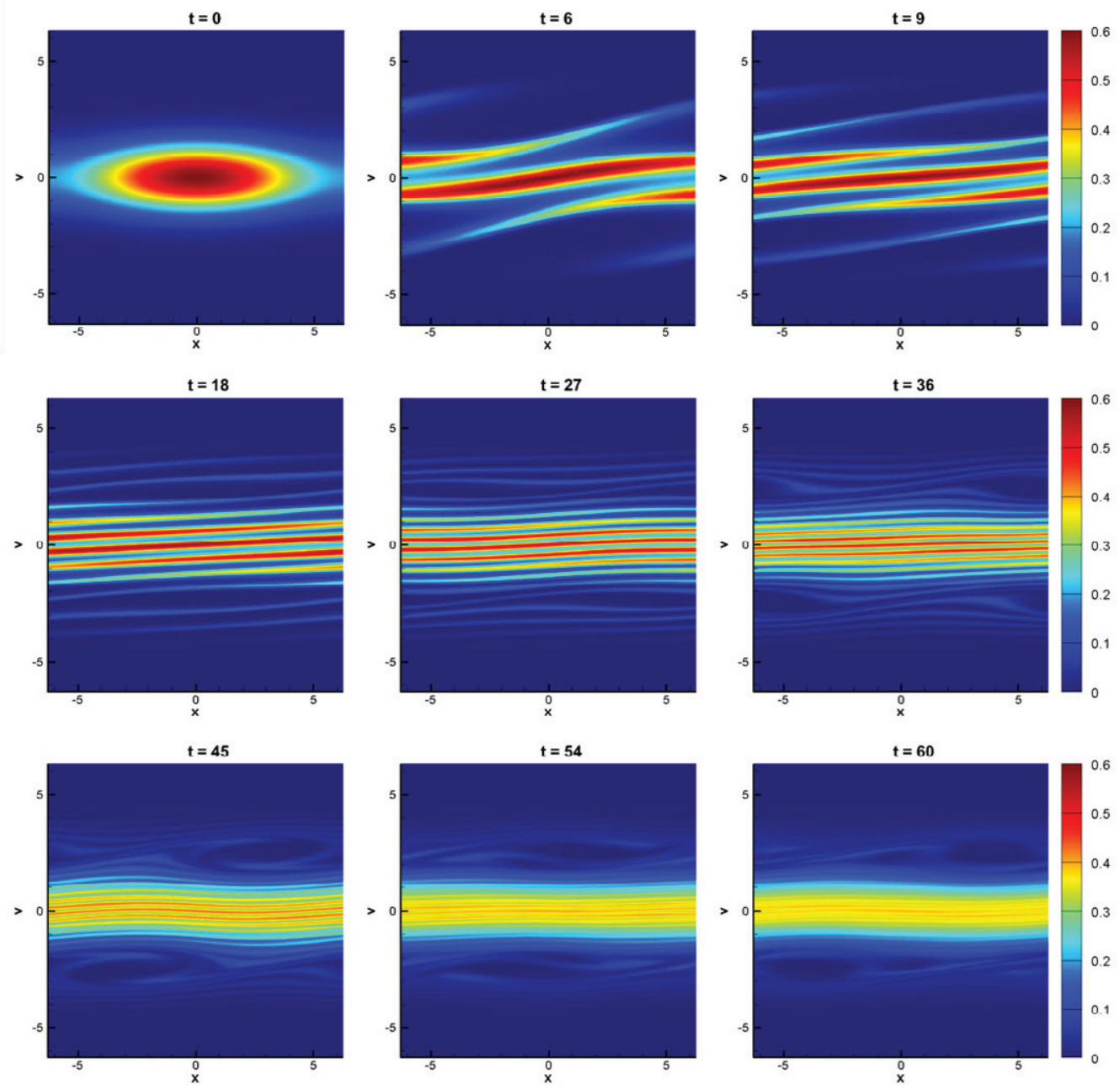

Figure 10: Time evolution of the phase space density for the strong Landau damping case with $\Delta t=1 / 8,256 \times 256$ particles, the 2-point quadrature rule, and 128 cells for the Poisson solve.

be compared with Fig. 5 in [4]. Figure 16 shows the error decreasing with increasing resolution and demonstrates that the method is second-order.

\section{A Note on Collisional Interactions}

In PIC codes collisional physics is typically included through binary collisions between the particles residing in the same spatial cell [29]. In the 

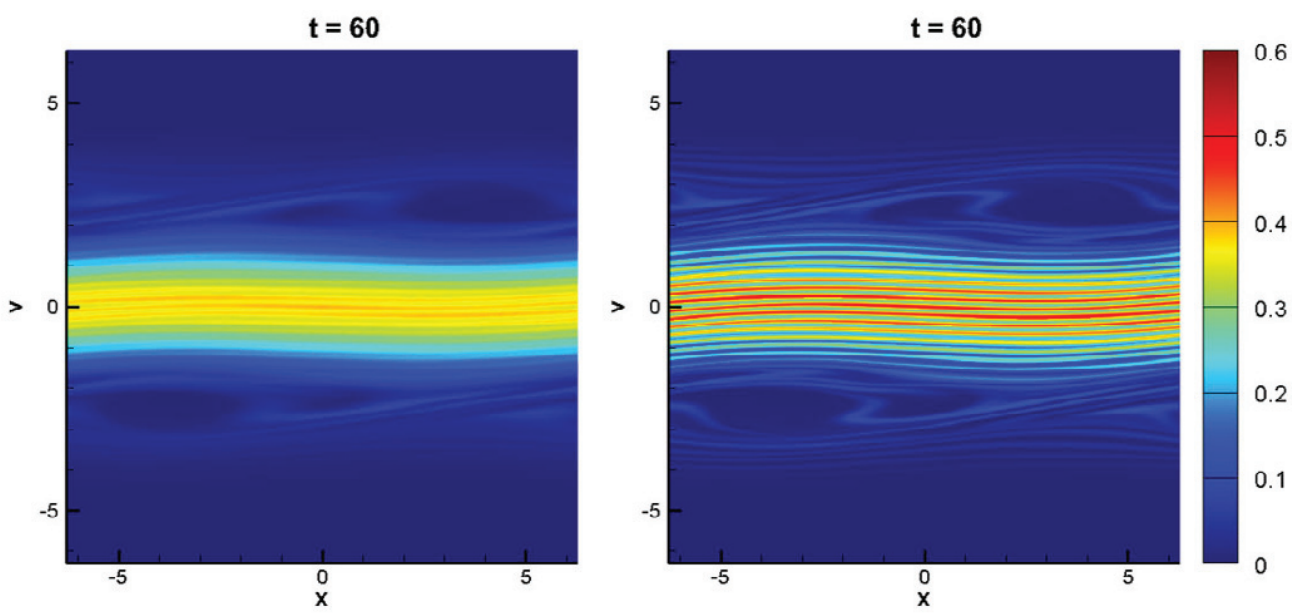

Figure 11: Final phase space density for the strong Landau damping case. The plot on the left corresponds to the final frame in Fig. 10. The plot on the right used $\Delta t=1 / 16$, $512 \times 512$ particles, and the 2-point quadrature rule.

SFK approach, the analogous process of pairing quadrature points that reside in the same cell using techniques appropriate for weighted particles $[30,31,32,33]$ could be used to add collisional physics. However, this would add noise to the dynamics due to the random scattering angles employed in each binary collision. The noise could be reduced, and perhaps eliminated, by employing the Fokker-Planck equation for Coulomb collisions [34] to calculate a velocity-space diffusion matrix for each interacting quadrature point, effectively increasing its phase-space volume from zero to a finite value in a fully deterministic way. The spread in velocity would then be included when calculating the covariance matrix to update the SFK particle envelope.

\section{Conclusions and Future Work}

We have described the shape function kinetics (SFK) method, based on the discrete partition of mass, for electrostatic plasma simulation. The method proceeds by advancing a set of quadrature nodes representing the mass distribution of the shape functions with standard PIC techniques. The updated positions and velocities are then used to reassemble the particle envelope via the center-of-mass coordinates and the covariance matrix. Test problems illustrate the advantages of the method, which include very low 


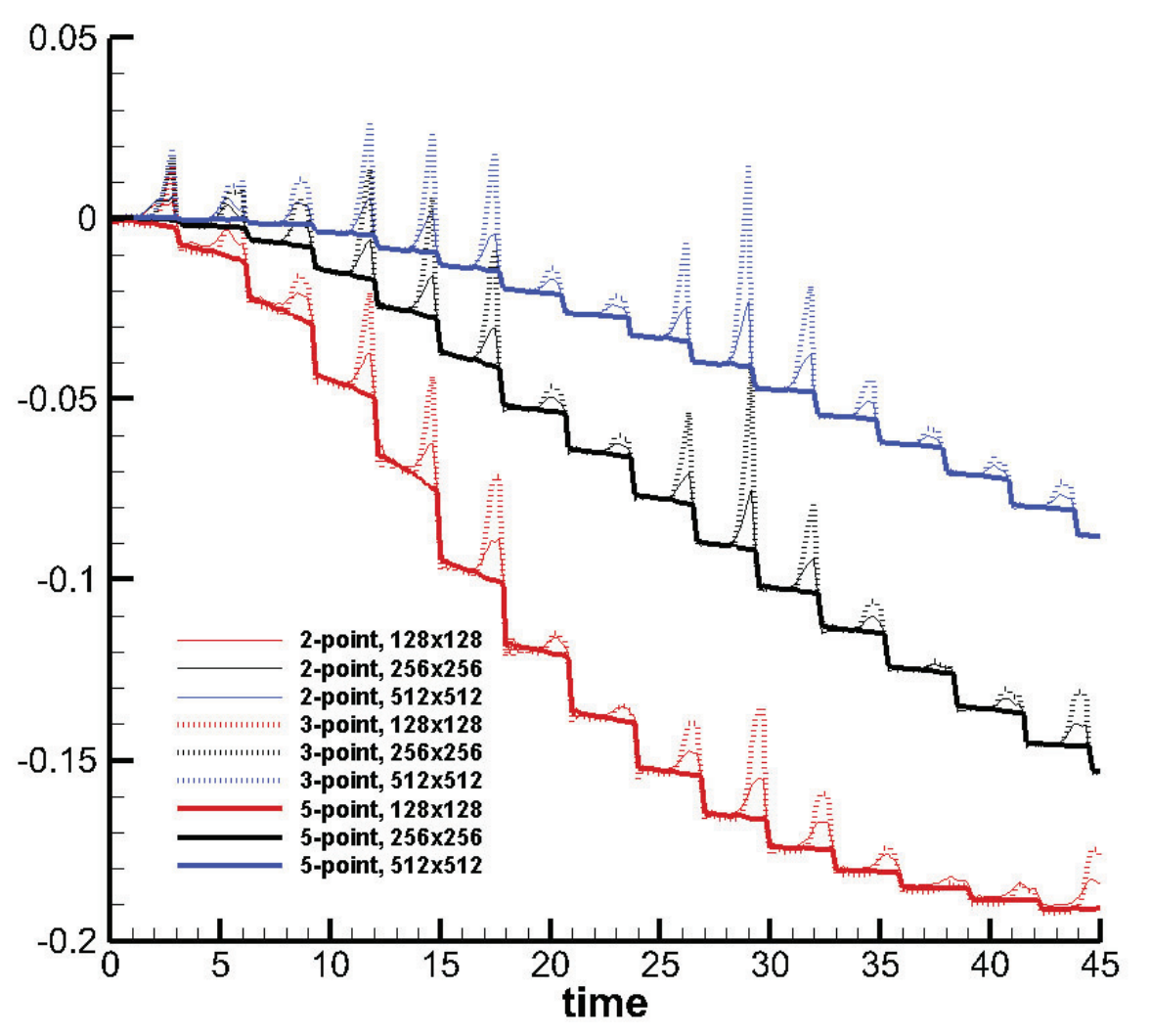

Figure 12: $L_{2}[f(t)]-L_{2}[f(t=0)]$ for the two-stream instability problem. The quadrature rule and the number of particles, e.g. $256 \times 256$, are indicated. All runs used $\Delta t=1 / 5$.

noise compared to the standard particle-in-cell technique, inherent positivity, large dynamic range, and ease of implementation.

Future work will focus on improving the remapping technique and including collisional interactions. Since all knowledge of the distribution function is carried by the particles between periodic remappings, it is conceivable that alternative remapping strategies, such as adaptive mesh refinement (AMR) or a fast Gaussian mixture model would greatly reduce the memory required for simulations in higher dimensions compared to fully mesh-based algorithms. The inclusion of collisions, via PIC-like interaction between the quadrature nodes, should retain the low-noise characteristics of the method by exploiting 


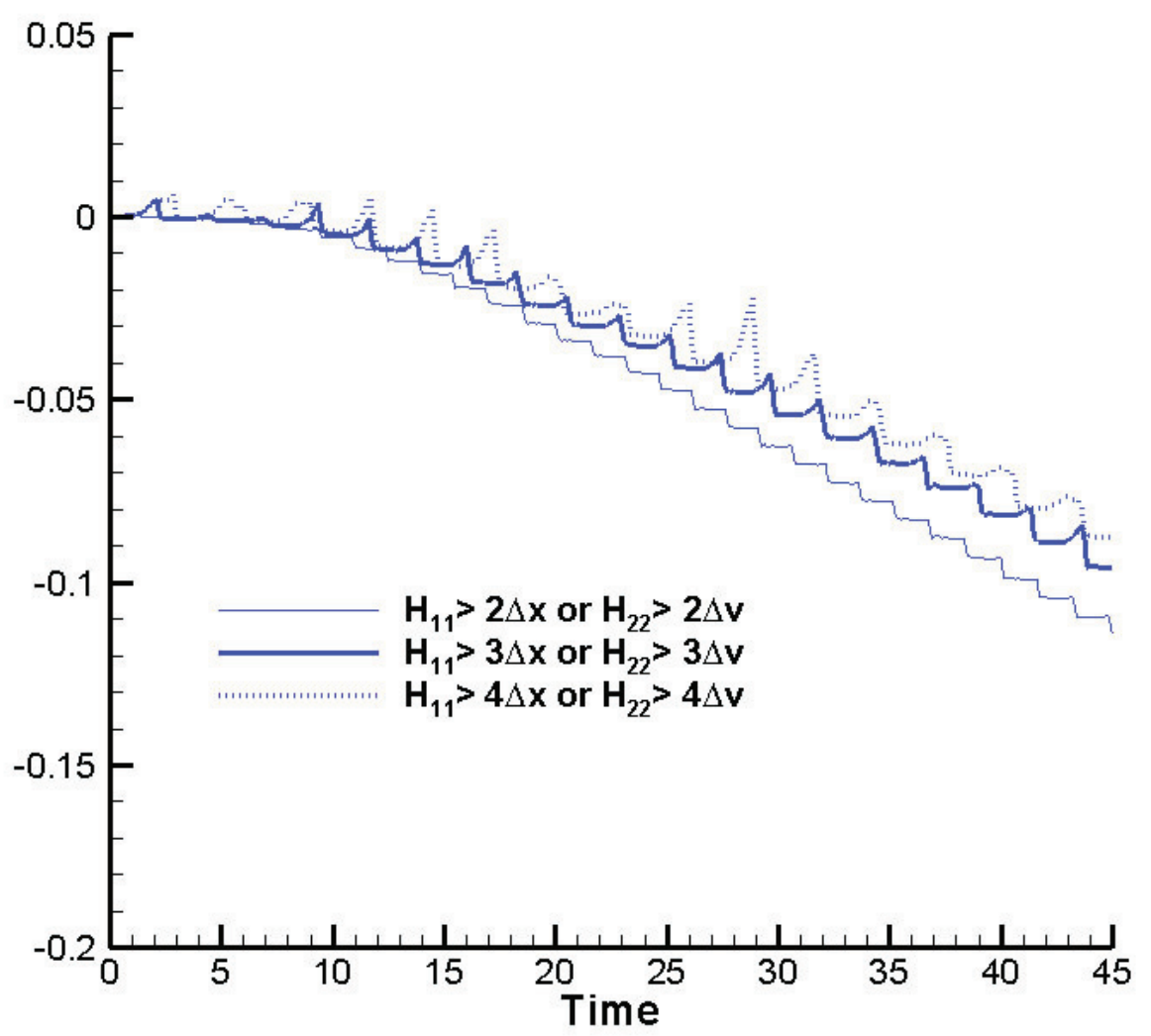

Figure 13: $L_{2}[f(t)]-L_{2}[f(t=0)]$ for the two-stream instability problem when the remapping frequency is varied. The simulations used $512 \times 512$ particles, the 2-point quadrature rule, 128 Poisson cells, and $\Delta t=1 / 8$.

the velocity variance carried by the particles.

\section{Acknowledgements}

The authors would like to acknowledge extensive discussions with the late Dennis Hewett. This work was performed under the auspices of the U.S. Department of Energy by Lawrence Livermore National Laboratory under Contract DE-AC52-07NA27344. C. V. Young acknowledges support from the DOE NNSA Stewardship Science Graduate Fellowship under contract DE-FC52-08NA28752. LLNL-JRNL-654126. 

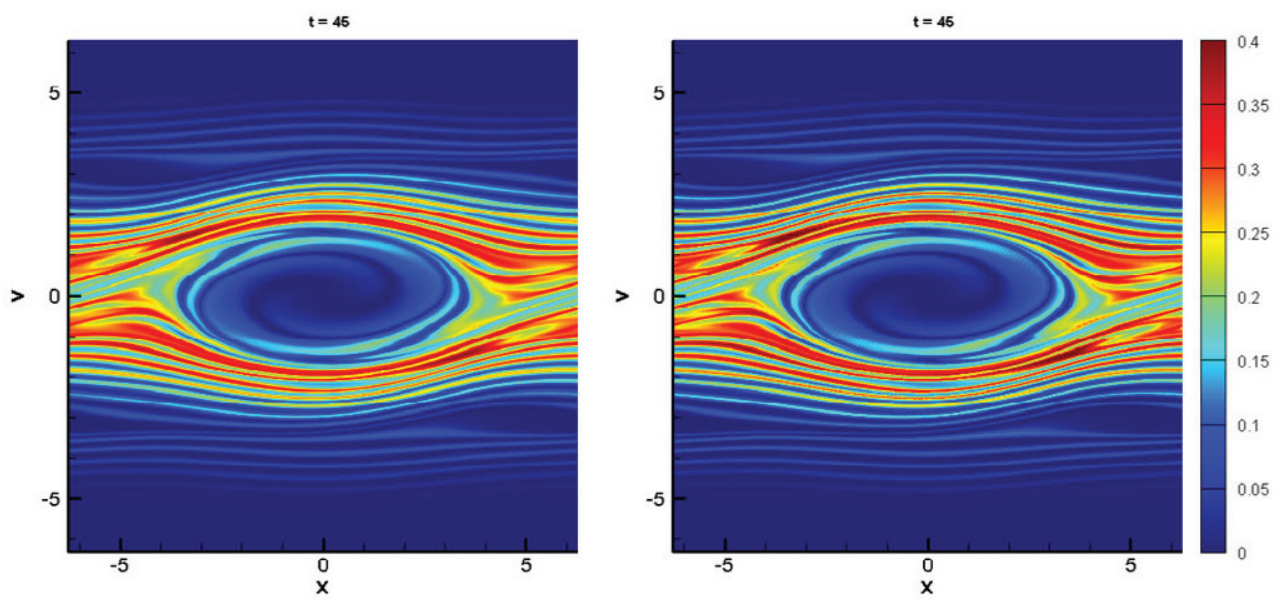

Figure 14: Phase space density for the two-stream instability problem at $t=45$ using $512 \times 512$ particles, the 2 -point quadrature rule, 128 Poisson cells, and $\Delta t=1 / 8$. The run plotted on the left remapped when $H_{11} \geq 2 \Delta x$ or $H_{22} \geq 2 \Delta v$ with a run time of 125 seconds. The run plotted on the right remapped when $H_{11} \geq 4 \Delta x$ or $H_{22} \geq 4 \Delta v$ with a run time of 94.4 seconds.

\section{References}

[1] C. K. Birdsall, A. B. Langdon, Plasma Physics Via Computer Simulation, New York: McGraw-Hill, 1985.

[2] R. W. Hockney, J. W. Eastwood, Computer Simulation Using Particles, Bristol: Adam Hilger, 1988.

[3] N. Crouseilles, M. Mehrenberger, E. Sonnendrucker, Conservative semilagrangian schemes for vlasov equations, Journal of Computational Physics 229 (2010) 1927-1953. doi:10.1016/j.jcp.2009.11.007.

[4] J. A. Rossmanith, D. C. Seal, A positivity-preserving high-order semilagrangian discontinuous galerkin scheme for the vlasov-poisson equations, Journal of Computational Physics 230 (2011) 6203-6232. doi: $10.1016 / j \cdot j c p .2011 .04 .018$.

[5] J.-M. Qiu, C.-W. Shu, Positivity preserving semi-lagrangian discontinuous galerkin formulation: theoretical analysis and application to the 


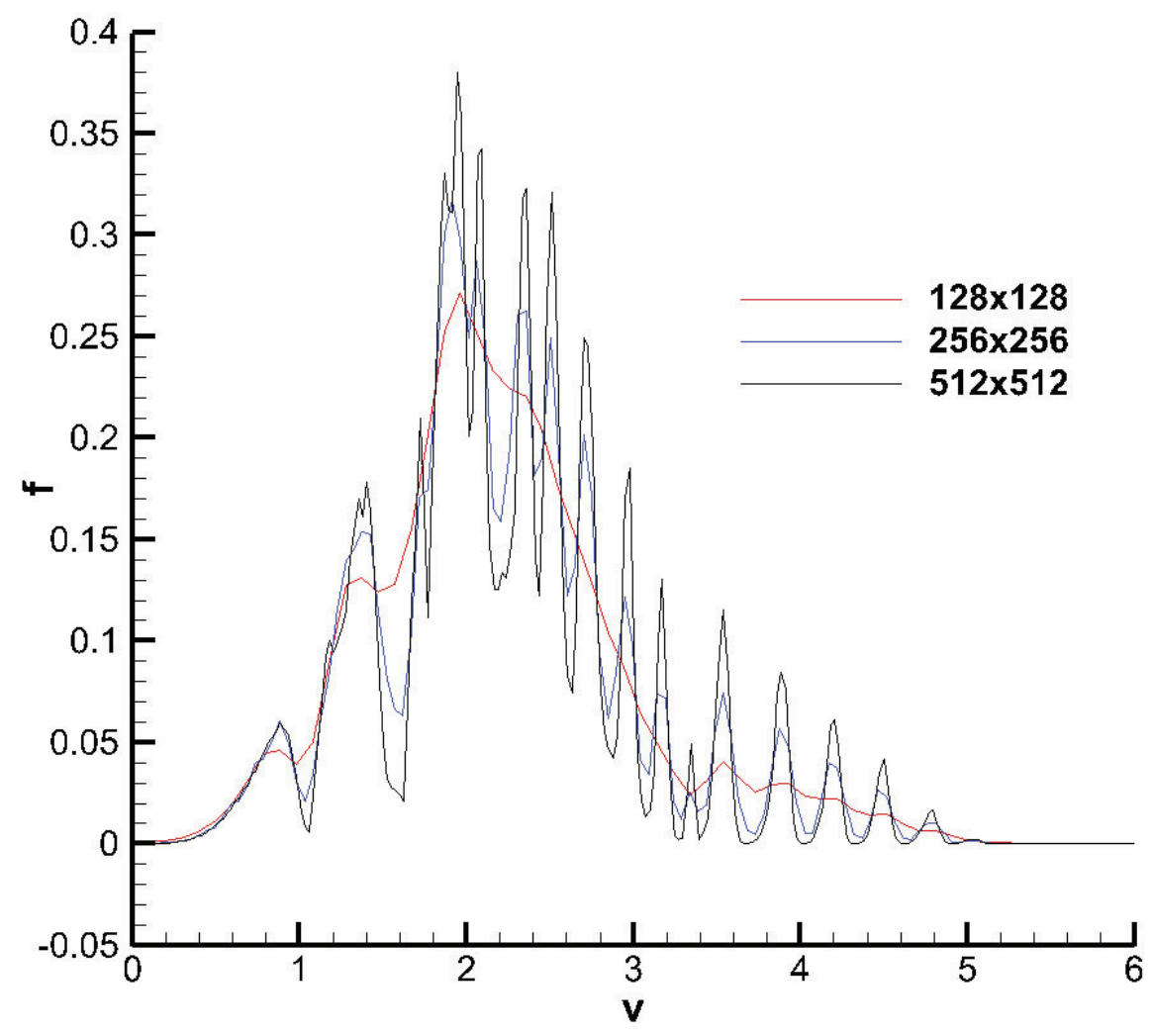

Figure 15: $f(x=0, v \geq 0)$ at $t=45$ for the two-stream instability problem. All runs used the 2-point quadrature rule and the remapping criteria $H_{11} \geq 4 \Delta x$ or $H_{22} \geq 4 \Delta v$.

vlasov-poisson system, Journal of Computational Physics 230 (2011) 8386-8409. doi:10.1016/j.jcp.2011.07.018.

[6] N. V. Elkina, J. Buchner, A new conservative unsplit method for the solution of the vlasov equation, Journal of Computational Physics 213 (2006) 862-875. doi:10.1016/j.jcp.2005.09.023.

[7] J. W. Banks, J. A. Hittinger, A new class of nonlinear finite-volume methods for vlasov simulation, IEEE Transactions on Plasma Science 38 (9) (2010) 2198-2207. doi:10.1109/TPS.2010.2056937. 

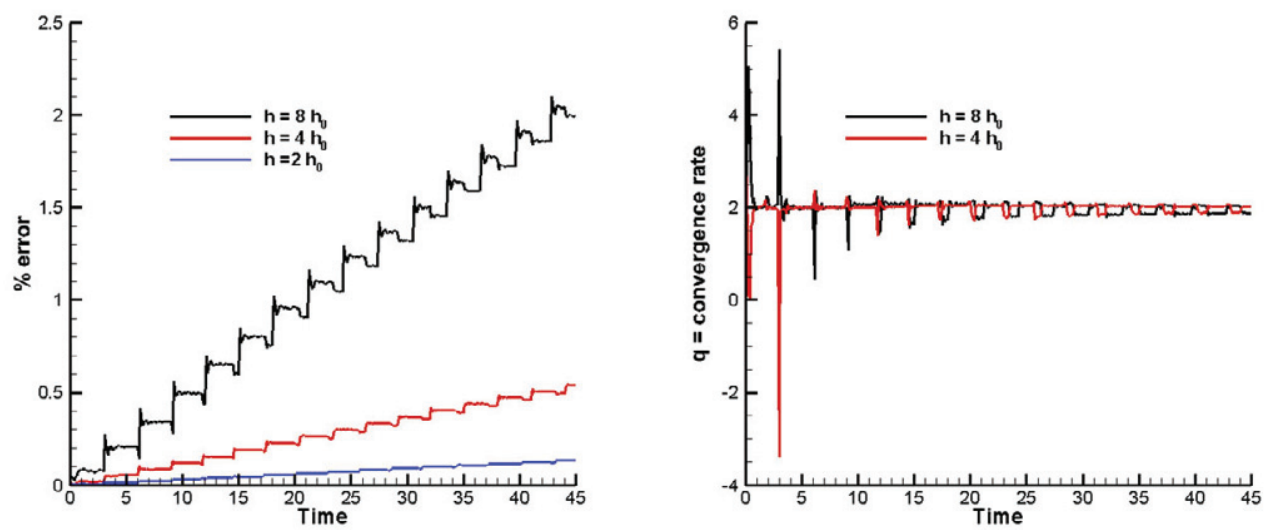

Figure 16: (a) Relative error in the total energy for the two-stream instability problem computed with increasing resolution. (b) Convergence rates for the errors in (a). The finest resolution is denoted by $h_{0}$ and was computed with $512 \times 512$ particles, 256 Poisson cells, and $\Delta t=1 / 64$.

[8] W. B. Bateson, D. W. Hewett, Grid and particle hydrodynamics, Journal of Computational Physics 144 (1998) 358-378. doi:10.1006/jcph. 1997.5824.

[9] D. W. Hewett, Fragmentation, merging, and internal dynamics for pic simulation with finite size particles, Journal of Computational Physics 189 (2003) 390-426. doi:10.1016/S0021-9991(03)00225-0.

[10] G. G. Coppa, V. Riccardo, G. Lapenta, Kinetic theory of charged particles of variable shape, Physics of Plasmas 3 (6) (1996) 2229-2238. doi:10.1063/1.871930.

[11] V. Riccardo, G. G. Coppa, G. Lapenta, Spices1 - a smart-particle code for kinetic plasma simulation, Computer Physics Communications 113 (1998) 199-219. doi:10.1016/S0010-4655(98)00075-7.

[12] C. Alard, S. Colombi, A cloudy vlasov solution, Monthly Notices of the Royal Astronomical Society 359 (2005) 123-163. doi:10.1111/j. 1365-2966.2005.08889.x.

[13] L. B. Lucy, An iterative techique for the rectification of observed distributions, The Astrophysical Journal 79 (6) (1974) 745-754. 
[14] M. C. Pinto, E. Sonnendrucker, A. Friedman, D. Grote, S. Lund, Noiseless vlasov-poisson simulations with linearly transformed particles, Journal of Computational Physics 275 (2014) 236-256. doi: 10.1016/j.jcp.2014.06.032.

[15] M. C. Pinto, Smooth particles without smoothing, arXiv: 112.1859v2 [math.NA].

[16] C. Gauger, P. Leinen, H. Yserentant, The finite mass method, SIAM Journal of Numerical Analysis 37 (6) (2000) 1768-799. doi:10.1137/ S0036142999352564.

[17] H. Yserentant, A new class of particle methods, Numerische Mathematik 76 (1997) 87-109. doi:10.1007/s002110050255.

[18] H. Yserentant, A particle model of compressible fluids, Numerische Mathematik 76 (1997) 87-109. doi:10.1007/s002110050256.

[19] H. Yserentant, Particles of variable size, Numerische Mathematik 82 (1999) 143-159. doi:10.1007/s002110050414.

[20] H. Yserentant, Entropy generation and shock resolution in the particle model of compressible fluids, Numerische Mathematik 82 (1999) 161177. doi:10.1007/s002110050415.

[21] H. Yserentant, The convergence of the finite mass method for flows in given force and velocity fields, in: M. Griebel, M. A. Schweitzer (Eds.), Meshfree Methods for Partial Differential Equations, Lecture Notes in Computational Science and Engineering, Vol. 26, Springer Berlin Heidelberg, 2003, pp. 419-440.

[22] B. Wang, G. H. Miller, P. Collela, A particle-in-cell method with adaptive phase-space remapping for kinetic plasmas, SIAM Journal of Scientific Computing 33 (2011) 3509-3537. doi:10.1137/100811805.

[23] M. Klingler, P. Leinen, H. Yserentant, A restart procedure for the finite mass method, SIAM Journal of Scientific Computing 30 (1) (2007) 117133. doi:10.1137/050641235.

[24] P. Li, H. Johnston, R. Krasny, A cartesian treecode for screened coulomb interactions, Journal of Computational Physics 228 (2009) 3858-3868. doi:10.1016/j.jcp.2009.02.022. 
[25] W. H. Press, S. A. Teukolsky, W. T. Vetterling, B. P. Flannery, Numerical Recipes The Art of Scientific Computing, 3rd Edition, New York: Cambridge University Press, 2007.

[26] A. Moore, Very fast em-based mixure model clustering using multiresolution kd-trees, in: Neural Information Processing Systems Conference, 1998.

[27] D. Xiu, Fast numerical methods for stochastic computations: a review, Communications in Computational Physics 5 (2-4) (2009) 242-272.

[28] D. Xiu, Numerical integration formulas of degree two, Applied Numerical Mathematics 58 (2008) 1515-1520. doi:10.1016/j.apnum. 2007. 09.004 .

[29] T. Takizuka, H. Abe, A binary collision model for plasma simulation with a particle code, Journal of Computational Physics 25 (1977) 205219. doi:10.1016/0021-9991(77)90099-7.

[30] K. Nanbu, S. Yonemura, Weighted particles in coulomb collision simulations based on the theory of a cumulative scattering angle, Journal of Computational Physics 145 (1998) 639-654. doi:10.1006/jcph.1998. 6049 .

[31] D. S. Lemons, D. Winske, W. Daughton, B. Albright, Small-angle coulomb collision model for particle-in-cell simulations, Journal of Computational Physics 228 (2009) 1391-1403. doi:10.1016/j.jcp. 2008. 10.025 .

[32] B. I. Cohen, A. M. Dimits, D. J. Strozzi, A grid-based binary model for coulomb collisions in plasmas, Journal of Computational Physics 234 (2013) 33-43. doi:10.1016/j.jcp.2012.08.046.

[33] R. H. Miller, M. R. Combi, A coulomb collision algorithm for weighted particle simulations, Geophysical Research Letters 21 (16) (1994) 17351738. doi:10.1029/94GL01835.

[34] M. N. Rosenbluth, W. M. MacDonald, D. L. Judd, Fokker-planck equation for an inverse-square force, Physical Review 107 (1) (1957) 1-6. doi:10.1103/PhysRev.107.1. 